\title{
Efeito da adubação fosfatada em Pinus taeda L. em Latossolo Vermelho na região de Telêmaco Borba, Paraná, Brasil ${ }^{1}$
}

\author{
James Stahl²; Alessandro Camargo Angelo ${ }^{3}$; Mauro Valdir Schumacher ${ }^{4}$; Karen Koch Fernandes de \\ Souza ${ }^{5}$; Fabricio Antonio Biernaski ${ }^{6}$
}

Resumo: Atualmente o Pinus taeda é a espécie de maior importância utilizada em reflorestamentos na região Sul do Brasil servindo de base para a cadeia produtiva do setor madeireiro. O objetivo deste trabalho foi avaliar aos dois e sete anos de idade a resposta de Pinus taeda a adição de doses de fósforo logo após o plantio em Latossolo Vermelho de textura argilosa e média na região de Telêmaco Borba, Paraná. Foram instalados dois experimentos de campo, um no solo de textura argilosa e o outro em textura média, em delineamento de blocos ao acaso, com quatro repetições, no qual os tratamentos consistiram em doses crescentes de fósforo $\left(0,50,75\right.$ e $\left.100 \mathrm{~kg} \mathrm{ha}^{-1} \mathrm{de}_{2} \mathrm{O}_{5}\right)$. As doses de fósforo foram aplicadas na forma de superfosfato triplo $\left(46 \% \mathrm{P}_{2} \mathrm{O}_{5}\right)$ em duas covetas laterais aos 30 dias após o plantio. $\mathrm{O}$ teor de fósforo disponível no solo foi caracterizado como muito baixo. Aos dois e sete anos foram medidos o diâmetro e altura total de 20 plantas por parcela e calculado o volume por área. Os resultados indicaram que o uso de adubação fosfatada promoveu ganhos significativos na produtividade de Pinus taeda até sete anos de idade no Latossolo Vermelho de textura média, e a resposta se deu de forma linear com

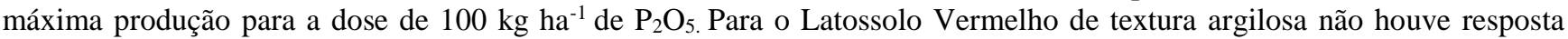
significativa ao uso de adubação fosfatada até os sete anos de idade.

Palavras-chave: Pinus; Nutrição Florestal; Silvicultura.

\section{Effect of phosphorus fertilization on Pinus taeda L. in Red Oxisol in the region of Telêmaco Borba, Paraná, Brasil}

\begin{abstract}
Currently the Pinus taeda is the most important species used in forest plantation in the southern region of Brazil, serving as the basis for the timber production chain. The purpose of this study was to evaluate the effect of phosphorus addition to the soil in Pinus taeda plantation at two and seven year olds after planting in clay and sandy clay Red Oxisol in the region of Telêmaco Borba, Paraná. Two field experiments were carried out, one in clay soil and the other in sandy clay soil, in a randomized block design with four replications, in which treatments consisted of increasing doses of phosphorus $(0,50,75$ and $\left.100 \mathrm{~kg} \mathrm{ha}^{-1} \mathrm{P}_{2} \mathrm{O}_{5}\right)$. The doses of phosphorus were applied in the form of triple superphosphate $\left(46 \% \mathrm{P}_{2} \mathrm{O}_{5}\right)$ in two lateral hole at 30 days after planting. The available soil phosphorus content was characterized as very low. At two and seven years, the diameter and total height of 20 plants per plot were measured, and volume was calculated by area. The results indicated that the use of phosphate fertilization promoted significant gains in the productivity of Pinus taeda up to seven years in sandy clay Red Oxisol, and the response was linear with maximum production for the dose of $100 \mathrm{~kg} \mathrm{ha}^{-1} \mathrm{P}_{2} \mathrm{O}_{5}$. For clay Red Oxisol there was no significant response to the use of phosphate fertilization until seven years of age.
\end{abstract}

Keywords: Pinus; Forest Nutrition; Silviculture.

\footnotetext{
${ }^{1}$ Recebido em 26.09.2017 e aceito para publicação como artigo científico em 18.11.2017.

2 Engenheiro Florestal, M.Sc., Doutorando do Programa de Pós-graduação em Engenharia Florestal, Universidade Federal do Paraná, Pesquisador na Klabin S.A. E-mail: <jstahl@klabin.com.br>.

3 Engenheiro Florestal, Dr.; Professor Associado do Departamento Ciências Florestais, Universidade Federal do Paraná. Email: <alessandrocangelo@gmail.com>.

${ }^{4}$ Engenheiro Florestal, Dr. nat. techn., Professor Titular do Departamento de Ciências Florestais, Universidade Federal de Santa Maria. Email: <mauro.schumacher@ufsm.br>.

5 Engenheira Florestal, Dr., Professora do Curso de Engenharia Florestal, Pontifícia Universidade Católica do Paraná. E-mail: $<$ karenkfs@yahoo.com.br>.

${ }^{6}$ Engenheiro Florestal, M.Sc., Doutorando do Programa de Pós-graduação em Engenharia Florestal, Universidade Federal do Paraná, Pesquisador na Klabin S.A. E-mail: <fbiernaski@klabin.com.br>.
} 


\section{Introdução}

O Pinus taeda L. é uma espécie subtropical proveniente do Sul dos Estados Unidos, ocorre naturalmente em 15 estados, abrangendo desde Delaware até a Flórida e o leste do Texas (MUNHOZ, 2011). Segundo Schultz (1997), é uma das espécies mais resistente e versátil devido à facilidade de reprodução e rápido crescimento em diferentes locais, o que a faz ser a principal espécie madeireira do sudeste dos Estados Unidos.

O plantio em larga escala no Brasil da espécie Pinus taeda teve início nos anos de 1960, pelos incentivos fiscais para reflorestamentos (KRONKA et al., 2005). Atualmente, a área plantada com Pinus no Brasil é de 1.584.332 hectares, concentra-se principalmente na Região Sul do país $(88,5 \%)$, devido às condições edafoclimáticas e à localização dos principais centros processadores desse tipo de madeira (IBÁ, 2017). O Estado do Paraná possui a maior área plantada de Pinus com 42,6\% da área total, seguido por Santa Catarina, que possui $34,5 \%$ (IBÁ, 2017).

Para Booth e Jovanovic (2000), o Pinus taeda pode ser plantado em regiões tropicais e subtropicais com altitudes entre 0 e $900 \mathrm{~m}$, precipitação média anual entre $900 \mathrm{~mm}$ e 2.200 mm e estação seca de 0 a 6 meses; temperatura média anual entre $14{ }^{\circ} \mathrm{C}$ e $24^{\circ} \mathrm{C}$ e temperatura média do mês mais quente entre $20^{\circ} \mathrm{C}$ e $35^{\circ} \mathrm{C}$, temperatura média do mês mais frio entre $1{ }^{\circ} \mathrm{C}$ e $18{ }^{\circ} \mathrm{C}$. É tolerante a geadas, podendo suportar períodos de alagamento do solo, bem como déficit hídrico. Embora apresente grande plasticidade, existe também grande variação de produtividade em função das condições edafoclimáticas, assim cresce em uma grande variedade de solos, de diferentes texturas, capacidade de retenção de umidade e acidez, mas apresenta baixa produtividade em solos de baixa fertilidade. De acordo com Higa et al. (2008), no estudo de zoneamento climático para Pinus taeda, a região Sul do Brasil praticamente não apresenta nenhuma condição restritiva ao desenvolvimento da espécie, como aquelas observadas na sua região de origem, como déficit hídrico e baixas temperaturas ao norte.

O Pinus taeda é uma espécie considerada de baixa exigência nutricional (PRITCHETT; ZWINFORD, 1961), a ausência de sintomas de deficiências, particularmente nas primeiras rotações, propiciou a ideia de que a espécie, assim como o gênero Pinus, dispensaria a prática de fertilização mineral. Reissmann e Wisniewski (2005), relatam que o pinus possui uma capacidade extraordinária de aproveitamento dos recursos nutricionais em sítios de baixa fertilidade, entretanto pode ocorrer diminuição do crescimento.

Durante quatro décadas de silvicultura de pinus no Brasil alguns experimentos e estudos de caso abordam o uso e resposta da espécie à adubação. Os trabalhos publicados no Brasil, concluem que o pinus, de uma forma geral responde menos à adubação que o eucalipto, mas que incrementos em volume podem chegar a $20 \%$ ou mais, em solos de baixa fertilidade (FERREIRA et al., 2001). Para a região de origem do Pinus taeda, principalmente Sul e Sudeste dos Estados Unidos, muitos trabalhos já foram desenvolvidos e mostram aumento de produtividade com uso de adubação, tanto no estabelecimento dos plantios, como em povoamentos em meia rotação (FOX et al., 2007).

De acordo com Gonçalves (1995), a adubação se faz necessária devido ao fato que nem sempre o solo é capaz de fornecer todos os nutrientes que as plantas demandam para um adequado crescimento. Dessa forma, as características e quantidades de adubo a serem utilizados dependem das necessidades nutricionais de cada espécie. O mesmo autor recomenda para Pinus taeda em solos de baixa a média disponibilidade de fósforo no solo, dosagens que variam de 20 a $60 \mathrm{~kg} \mathrm{ha}^{-1}$ de $\mathrm{P}_{2} \mathrm{O}_{5}$. Belotte e Dedecek (2006), descrevem em seu trabalho que aumentos de produtividade em Pinus taeda podem ser obtidos com a adoção de diversas práticas silviculturais, tais como descompactação do solo, poda e desbastes, e dentre elas a fertilização.

O objetivo deste trabalho foi avaliar, aos dois e sete anos de idade, a resposta de Pinus taeda a adição de dose de fósforo logo após o plantio em 
Latossolo Vermelho de textura argilosa e média na região de Telêmaco Borba, Paraná.

\section{Material e métodos}

Os dados deste trabalho referem-se a dois experimentos de campo com Pinus taeda, um sobre Latossolo Vermelho textura argilosa (Experimento I, 50³2’41" W; 241' $30^{\circ}$ ” S), e outro em Latossolo Vermelho textura média (Experimento II, 50³0’01” W; 2416’50” S), implantados na fazenda Monte Alegre da empresa Klabin S.A, no município de Telêmaco Borba, Paraná.

As duas áreas experimentais apresentavam características de manejo semelhantes na rotação anterior, plantio de Pinus taeda com corte final aos 18 anos. $\mathrm{O}$ solo foi coletado na camada 0 20 e $20-40 \mathrm{~cm}$ (Tabela 1) e analisado de acordo com a metodologia de Tedesco et al. (1995), para as duas áreas experimentais antes do início da implantação do experimento. Os teores de fósforo disponíveis (extraídos pelo método Melich-1) no solo (Tabela 1) são classificados como muito baixos para espécies florestais (GATIBONI et al., 2016), tanto para a área experimental instalada sobre Latossolo Vermelho textura argilosa, quanto para a área experimental instalada sobre Latossolo Vermelho textura média.

As técnicas de manejo utilizadas na implantação e condução dos dois experimentos foram as mesmas. Nas duas áreas foi realizado preparo do solo mecanizado em cultivo mínimo com subsolagem a $45 \mathrm{~cm}$ de profundidade e 60 $\mathrm{cm}$ de largura na linha de plantio. As plantas daninhas foram controladas na linha de plantio com herbicida pré-emergente, e nas entrelinhas com dessecante até o fechamento de copa.

Tabela 1 - Características químicas e físicas do solo nas camadas 0-20 e 20-40 cm na área experimental de Latossolo Vermelho textura argilosa e textura média.

Table 1 - Chemical and physical characteristics of the soil in layers 0-20 and 20-40 cm in the experimental area in clay and sandy clay Red Oxisol.

\begin{tabular}{|c|c|c|c|c|c|}
\hline \multirow{3}{*}{ Atributos } & \multirow{3}{*}{ Unidades } & \multicolumn{2}{|c|}{ Exp. I (Latossolo textura argilosa) } & \multicolumn{2}{|c|}{ Exp. II (Latossolo textura média) } \\
\hline & & \multicolumn{4}{|c|}{ Profundidade $(\mathrm{cm})$} \\
\hline & & $0-20$ & $20-40$ & $0-20$ & $20-40$ \\
\hline Areia Grossa & \multirow{5}{*}{$(\%)$} & 4 & 5 & 16 & 15 \\
\hline Areia Fina & & 10 & 9 & 54 & 52 \\
\hline Silte & & 33 & 30 & 10 & 11 \\
\hline Argila & & 53 & 55 & 20 & 22 \\
\hline M.O. & & 3,4 & 2,5 & 1,1 & 0,8 \\
\hline $\mathrm{pH}-\mathrm{H}_{2} \mathrm{O}$ & & 4,5 & 4,7 & 4,4 & 4,5 \\
\hline $\mathrm{P}$ & \multirow{2}{*}{$\left(\mathrm{mg} \mathrm{dm}^{-3}\right)$} & 1,7 & 1,1 & 1,1 & 0,7 \\
\hline $\mathrm{K}$ & & 31 & 21 & 15 & 10 \\
\hline $\mathrm{Al}^{3+}$ & \multirow{3}{*}{$\left(\mathrm{cmol}_{\mathrm{c}} \mathrm{dm}^{-3}\right)$} & 2,1 & 2,2 & 2,4 & 2,2 \\
\hline $\mathrm{Ca}^{2+}$ & & 1,5 & 0,4 & 0,1 & 0,1 \\
\hline $\mathrm{Mg}^{2+}$ & & 0,1 & 0,2 & 0,1 & 0,1 \\
\hline
\end{tabular}

Nos dois experimentos foram utilizadas mudas de origem seminal de pomar clonal de segunda geração. As mudas foram produzidas em Telêmaco Borba, PR, no viveiro operacional da Klabin, e apresentavam seis meses de idade na ocasião do plantio. O plantio foi realizado em 
maio/2007 com espaçamento de plantio de 2,5 m entre plantas e 2,5 $\mathrm{m}$ nas entrelinhas.

Os tratamentos de adubação foram os mesmos para os dois experimentos e consistiram em quatro doses de fósforo $\left(0,50,75,100 \mathrm{~kg} \mathrm{ha}^{-}\right.$ 1 de $\left.\mathrm{P}_{2} \mathrm{O}_{5}\right)$. Utilizou-se $\mathrm{o}$ delineamento experimental de blocos ao acaso com quatro repetições, sendo a unidade experimental constituída de uma parcela de 60 plantas $(3 \mathrm{x}$ 20), das quais somente as 20 plantas centrais foram utilizadas para as mensurações, com bordadura dupla em cada unidade. As doses de fósforo foram aplicadas na forma de superfosfato triplo $\left(46 \% \mathrm{P}_{2} \mathrm{O}_{5}\right)$ em duas covetas laterais, a $10 \mathrm{~cm}$ de distância da muda e $10 \mathrm{~cm}$ de profundidade, 30 dias após o plantio.

Aos dois anos foi mensurada a altura total e diâmetro a $50 \mathrm{~cm}$ do solo das plantas da parcela de mensuração. Já na idade de sete anos após o plantio foi mensurada altura total e o diâmetro a 1,30 $\mathrm{m}$ do solo (Dap). A partir dos dados de altura e diâmetro calculou-se o volume por hectare de cada parcela usando fator de forma de $0,45($ Volume $=($ g.h. $f))$, onde $\left.g=\left((\text { Dap })^{2} \cdot \pi\right) / 4\right)$; $f=$ fator de forma; e $\mathrm{h}=$ altura.

Os dados obtidos foram submetidos a análises estatísticas utilizando o aplicativo "Statistical Analysis System" (SAS, 2016). Procedeu-se a análise de variância (teste $\mathrm{F}$ ) e, teste de comparação de média de Tukey a significância de 5\% para altura e Dap. Para o volume efetuaram-se análises de regressão pelo procedimento PROREG para estudo dos tratamentos em que o efeito foi significativo no teste F.

\section{Resultados e Discussão}

As doses de fósforo não apresentaram diferenças estatisticamente significativas no arranque inicial de crescimento de Pinus taeda quando observado as variáveis diâmetro a $50 \mathrm{~cm}$ e altura (Tabela 2) aos dois anos de idades. Não houve diferença entre as doses dentro de cada experimento, e entre os experimentos na mesma dose.

Tabela 2 - Valores médios de diâmetro e altura (h) de Pinus taeda aos dois e sete anos de idade em função de doses de fósforo $\left(\mathrm{P}_{2} \mathrm{O}_{5}\right)$ em Latossolo Vermelho de textura argilosa e média.

Table 2 - Average values of diameter and height (h) of Pinus taeda at two and seven years of age as a function of doses of phosphorus $\left(\mathrm{P}_{2} \mathrm{O}_{5}\right)$ in a clay and sandy clay Red Oxisol.

\begin{tabular}{|c|c|c|c|c|}
\hline \multirow{3}{*}{$\begin{array}{c}\text { Doses de } \mathrm{P}_{2} \mathrm{O}_{5} \\
\left(\mathrm{~kg} \mathrm{ha}^{1}\right)\end{array}$} & \multicolumn{2}{|c|}{ 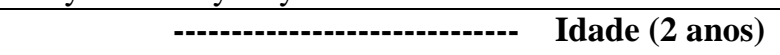 } & \multicolumn{2}{|c|}{ 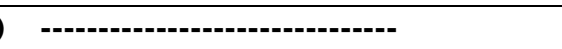 } \\
\hline & \multicolumn{2}{|c|}{ Diâmetro (cm) } & \multicolumn{2}{|c|}{$\mathbf{h}(\mathbf{m})$} \\
\hline & Textura argilosa & Textura média & Textura argilosa & Textura média \\
\hline 0 & $1,75 \mathrm{Aa}^{*}$ & $1,70 \mathrm{Aa}$ & 3,01 Aa & 2,99 Aa \\
\hline 50 & $1,80 \mathrm{Aa}$ & $1,80 \mathrm{Aa}$ & $3,08 \mathrm{Aa}$ & $3,15 \mathrm{Aa}$ \\
\hline 75 & $1,93 \mathrm{Aa}$ & $1,80 \mathrm{Aa}$ & $3,20 \mathrm{Aa}$ & $3,10 \mathrm{Aa}$ \\
\hline 100 & $1,88 \mathrm{Aa}$ & $1,86 \mathrm{Aa}$ & $3,19 \mathrm{Aa}$ & $3,19 \mathrm{Aa}$ \\
\hline \multirow[t]{2}{*}{$\mathrm{CV}(\%)$} & 7,86 & 9,67 & 5,30 & 6,81 \\
\hline & \multicolumn{3}{|c|}{ 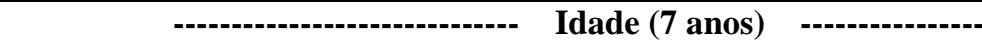 } & 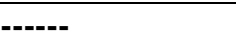 \\
\hline 0 & $18,75 \mathrm{Aa}$ & $17,47 \mathrm{Aa}$ & $11,83 \mathrm{Aa}$ & $10,08 \mathrm{Ba}$ \\
\hline 50 & $18,42 \mathrm{Aa}$ & $17,72 \mathrm{Aa}$ & $12,32 \mathrm{Aa}$ & $10,15 \mathrm{Ba}$ \\
\hline 75 & $18,54 \mathrm{Aa}$ & $18,38 \mathrm{Aa}$ & $12,32 \mathrm{Aa}$ & $10,09 \mathrm{Ba}$ \\
\hline 100 & $18,33 \mathrm{Aa}$ & $18,65 \mathrm{Aa}$ & $12,16 \mathrm{Aa}$ & $10,38 \mathrm{Ba}$ \\
\hline $\mathrm{CV}(\%)$ & 4,05 & 3,62 & 3,23 & 6,29 \\
\hline
\end{tabular}

* Médias seguidas pelas mesmas letras, maiúsculas nas linhas, e minúsculas nas colunas, não diferem estatisticamente entre si pelo Teste de Tukey a $5 \%$ de probabilidade. 
Em valores absolutos, observa-se aos dois anos uma resposta da ordem de 10,3\% em diâmetro quando comparado ao tratamento sem

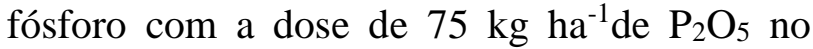
Latossolo de textura argilosa. Para essa mesma variável, no ambiente de textura média, o incremento em diâmetro foi de $9,4 \%$ da dose de $100 \mathrm{~kg} \mathrm{ha}^{-1} \mathrm{de} \mathrm{P}_{2} \mathrm{O}_{5}$, quando comparado ao tratamento sem fósforo. Para a variável $\mathrm{h}$ aos dois anos a resposta absoluta foi de $6,3 \%$ maior comparado ao tratamento sem fósforo com a dose de $75 \mathrm{~kg} \mathrm{ha}^{-1}$ de $\mathrm{P}_{2} \mathrm{O}_{5}$ no ambiente argiloso, e de $6,7 \%$ na dose de $100 \mathrm{~kg} \mathrm{ha}^{-1} \mathrm{de}_{2} \mathrm{P}_{2} \mathrm{O}_{5} \mathrm{em}$ relação ao tratamento sem fósforo no ambiente de textura média.

Vogel et al. (2005), encontraram reposta positiva máxima para altura total de plantas de Pinus taeda aos 19 meses com dose de $96,8 \mathrm{~kg}$ ha $^{-1}$ de $\mathrm{P}_{2} \mathrm{O}_{5}$ em Cambissolo Húmico argiloso, que apresentava $1,5 \mathrm{mg} \mathrm{\textrm {dm } ^ { - 3 }}$ de fósforo disponível no solo. Resultados de ganhos no crescimento inicial de Pinus taeda foram demonstrados também por Faustino et al. (2012), estudando resposta a fertilização de quatro diferentes famílias selecionadas por ganho em volume e qualidade de fuste, em que houve ganhos significativos em altura e diâmetro do colo aos oito e dezesseis meses de idade em resposta a adição de 96 gramas planta ${ }^{-1}$ de $_{2} \mathrm{P}_{5}$ em solo vermelho, argiloso e profundo.

$\mathrm{Na}$ idade de sete anos o Dap e altura (Tabela 2) não diferiram estatisticamente em relação as doses de fósforo, tanto na área experimental em Latossolo Vermelho de textura argilosa, quanto na área de textura média. Mesmo não apresentando diferença estatística observa-se incremento de Dap de 6,6\% quando utiliza-se a dose de $100 \mathrm{~kg}$ ha ${ }^{-1}$ de $\mathrm{P}_{2} \mathrm{O}_{5}$ em relação ao tratamento sem fósforo. Nesta idade foi observado diferença significativa quando comparado à variável altura entre os dois ambientes para todos os tratamentos. Com os dados de altura, mesmo que sejam dados na idade de meia rotação, pode-se inferir que existe uma clara diferença de sítio entre as áreas experimentais. Analisando as médias de tratamentos sem fertilização, a altura média no Latossolo Vermelho de textura argiloso é 17,4\% maior que no Latossolo Vermelho de textura média aos sete anos de idade, já para o Dap a diferença é de 7,4\% maior, respectivamente.

Rigatto et al. (2005), estudando a relação de produtividade de Pinus taeda e atributos químicos e físico de diferentes classes concluíram que os solos de texturas argilosa propiciaram maior produtividade quando analisados aso doze anos de idade. Ainda destacam a correlação positiva entre $\mathrm{pH}, \mathrm{K}$ e Ca $+\mathrm{Mg}$ com a produtividade, atributos este que no presente estudo apresentam-se em níveis maiores na área experimental de textura argilosa.

Para o volume das parcelas, em que se integra as variáveis Dap e altura, a análise de variância (Tabela 3) indica que o ajuste de modelo de regressão das doses crescentes de $\mathrm{P}_{2} \mathrm{O}_{5}$ não foi significativo para o Latossolo Vermelho textura argilosa, tanto na idade de dois anos quanto na idade de sete anos. Já para o Latossolo Vermelho de textura média o modelo teve significância de $(\mathrm{P}<0,05)$ aos dois anos e $(\mathrm{P}<0,10)$ aos sete anos de idade.

Tabela 3 -Análise de variância com valores de quadrado médio (QM) de volume total de Pinus taeda aos dois e sete anos de idade em função de doses de fósforo $\left(\mathrm{P}_{2} \mathrm{O}_{5}\right)$ em Latossolo Vermelho de textura argilosa e média.

Tabela 3 - Analysis of variance with mean square for total volume of Pinus taeda at two and seven years of age as a function of doses of phosphorus $\left(\mathrm{P}_{2} \mathrm{O}_{5}\right)$ in a clay and sandy clay Red Oxisol.

QM

\begin{tabular}{ccc}
\cline { 2 - 3 } Experimento & \multicolumn{2}{c}{ Fonte de Variação (Doses de $\left.\mathbf{P}_{2} \mathbf{O}_{5}\right)$} \\
\cline { 2 - 3 } & Idade $(\mathbf{2}$ anos) & Idade $(\mathbf{7}$ anos $)$ \\
\hline Textura argilosa & $0,07^{\mathrm{ns}}$ & $25,50^{\mathrm{ns}}$ \\
Textura média & $0,02^{*}$ & $610,44^{* *}$ \\
\hline
\end{tabular}

ns - não significativo; *significativo $(\mathrm{P}<0,05)$; **significativo $(\mathrm{P}<0,10)$. 
O volume de Pinus taeda apresentou comportamento linear (Figura 1) com o aumento das doses de $\mathrm{P}_{2} \mathrm{O}_{5}$ adicionadas ao solo aos dois anos de idade para o Latossolo Vermelho textura média. $\mathrm{O}$ volume inicial atingido com a dosagem de $100 \mathrm{~kg} \mathrm{ha}^{-1}$ de $\mathrm{P}_{2} \mathrm{O}_{5}$ foi $24,4 \%$ maior do que o volume no tratamento sem fósforo. Já no Latossolo Vermelho argiloso a maior média de volume foi para a dosagem de $75 \mathrm{~kg} \mathrm{ha}^{-1} \mathrm{de}_{2} \mathrm{O}_{5}$. Em solos argilosos na província de Missiones na Argentina, Fernandez et al. (2000), estudaram o efeito da aplicação de doses crescentes de fósforo, além de potássio e de nitrogênio, e constataram diferenças significativas no diâmetro, altura total e volume de Pinus taeda aos 34 meses, com máxima resposta relacionado à adição de $62 \mathrm{~kg} \mathrm{ha}{ }^{-1}$ de $\mathrm{P}_{2} \mathrm{O}_{5}$. Similar ao trabalho anterior Vogel et al. (2005), em que encontraram máxima resposta em volume cilíndrico de Pinus taeda em Cambissolo

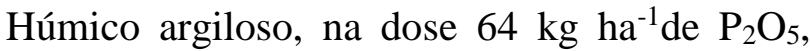
quando avaliado aos dezenove meses de idade.

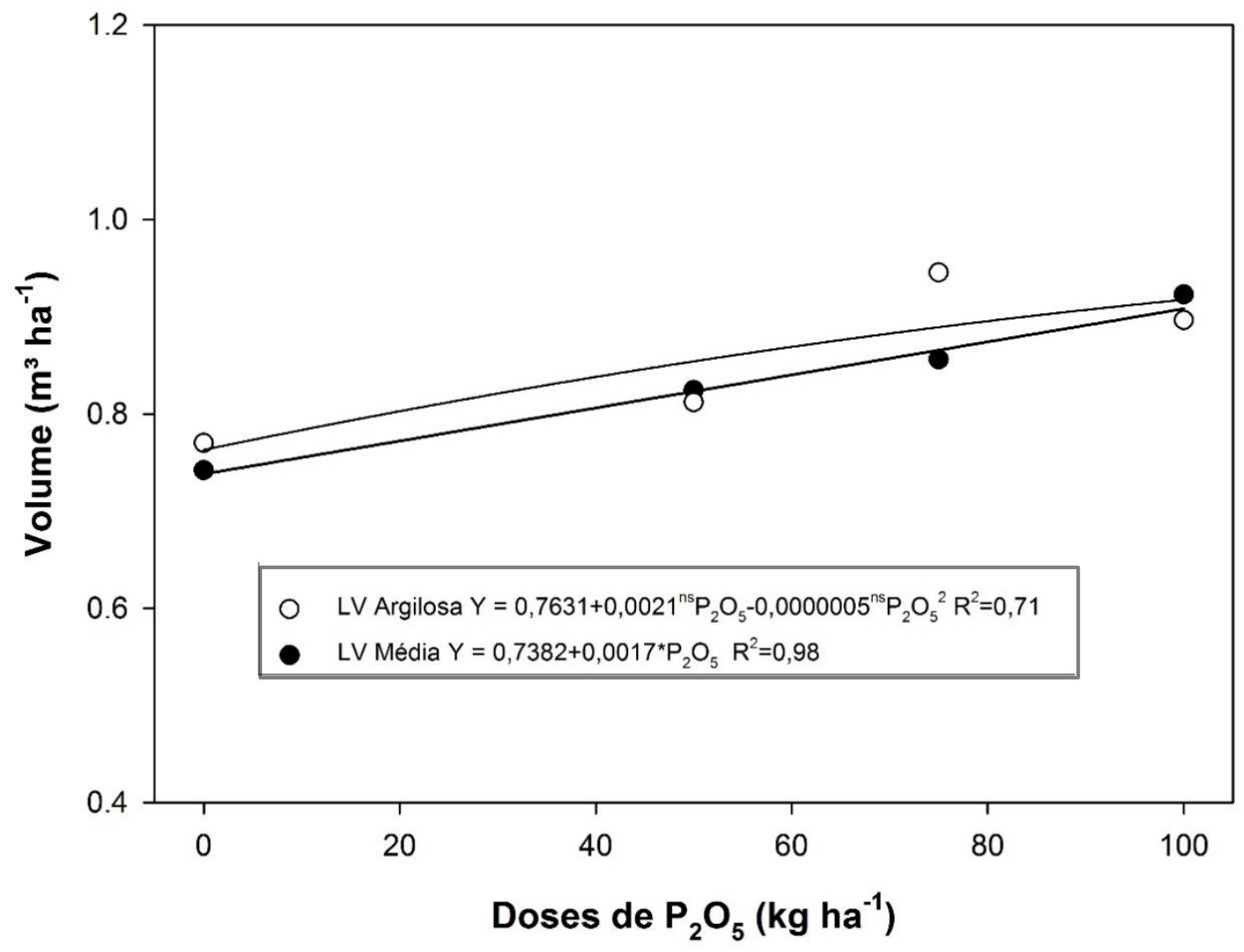

Figura 1 - Resposta em volume para Pinus taeda aos dois anos de idade, em função da adição de doses de fósforo, em Latassolo Vermelho de textura argilosa e média.

Figure 1 - Volume response for Pinus taeda at two years of age, as influenced by doses of phosphorus added in a clay and sandy clay Red Oxisol.

Os resultados em volume aos sete anos, após o uso da adubação com doses crescentes de fósforo, apontam diferenças entre os ambientes, experimento em textura argilosa e textura média, e o comportamento da curva de resposta. Nessa idade está praticamente caracterizado metade do ciclo de rotação, planejado para esses plantios com idade de quinze anos.

Aos sete anos manteve-se o mesmo comportamento de resposta linear bem definida da adição de doses crescentes de fósforo no Latossolo Vermelho de textura média (Figura 2). 
Neste ambiente o uso da dose $100 \mathrm{~kg} \mathrm{ha}^{-1} \mathrm{de}_{2} \mathrm{O}_{5}$ promoveu ganho em volume de $17,4 \%$ maior correspondendo a uma diferença de $33,4 \mathrm{~m}^{3} \mathrm{ha}^{-}$ que $o$ tratamento sem fertilização,

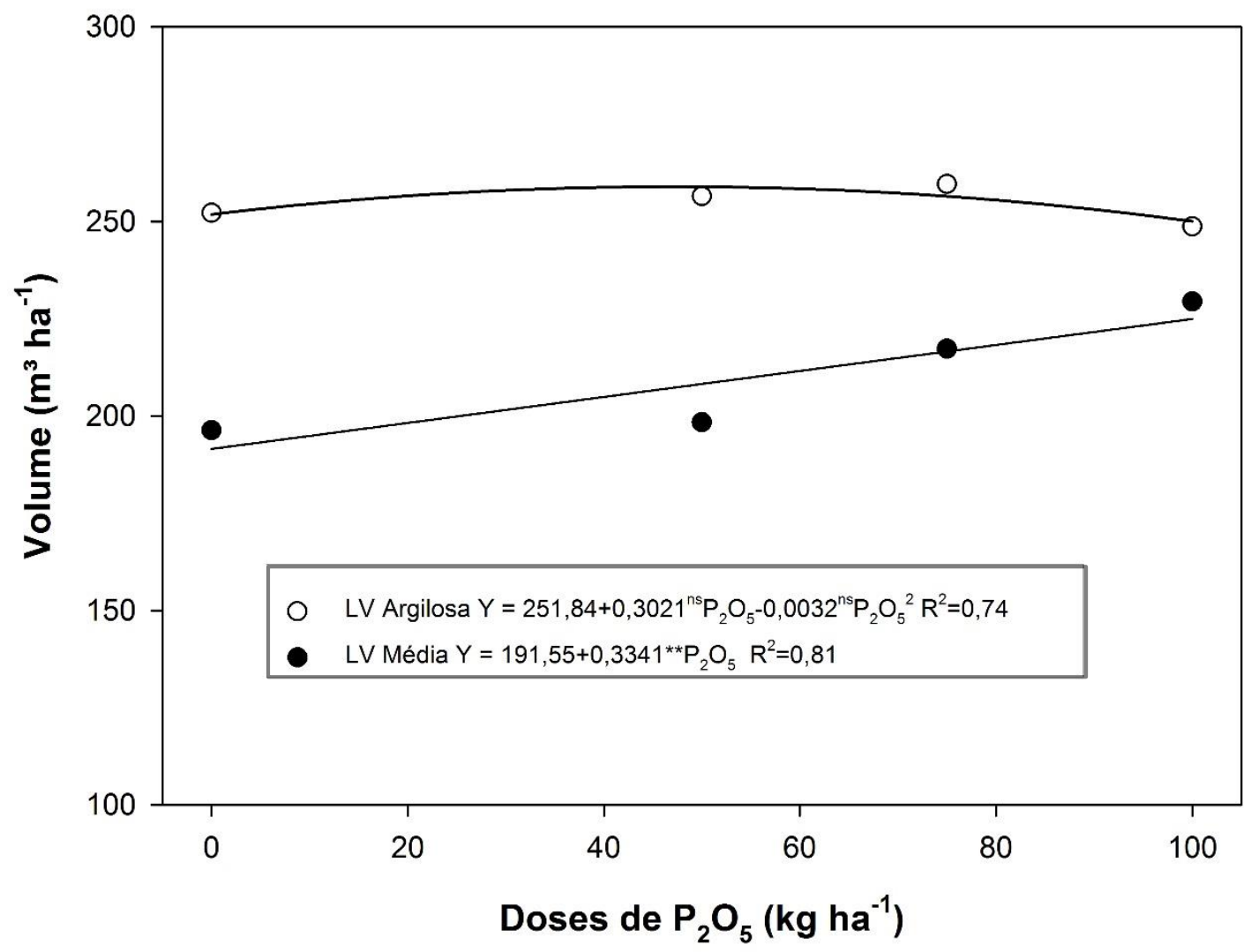

Figura 2 - Resposta em volume para Pinus taeda aos sete anos de idade, em função da adição de doses de fósforo, em Latassolo Vermelho de textura argilosa e média.

Figure 2 - Volume response for Pinus taeda at seven years of age, as influenced by doses of phosphorus added in a clay and sandy clay Red Oxisol.

O resultado de volume aos sete anos no Latossolo textura argilosa apresentou comportamento quadrático em relação as doses de fósforo, porém não teve efeito significativo. Em valores absolutos o volume médio, maior nesse ambiente, foi para a dose de $50 \mathrm{~kg} \mathrm{ha}^{-1} \mathrm{de}$ $\mathrm{P}_{2} \mathrm{O}_{5}$, correspondendo a um ganho de pequena magnitude de $2,9 \%$ em relação ao tratamento sem fósforo.

Analisando os resultados em volume aos sete anos fica clara a diferença de produtividade entre os ambientes, tomando-se como referência os tratamentos sem adição de fósforo, a produção em volume no Latossolo Vermelho de textura argilosa foi $31,5 \%$ maior em relação ao
Latossolo Vermelho de textura média, diferença que corresponde a $60,3 \mathrm{~m}^{3} \mathrm{ha}^{-1}$. Comparando as máximas produção em função das doses, de $258,9 \mathrm{~m}^{3} \mathrm{ha}^{-1}$ na dose de $50 \mathrm{~kg} \mathrm{ha}^{-1} \mathrm{de}_{2} \mathrm{O}_{5}$ para o a textura argilosa, e de $211,7 \mathrm{~m}^{3} \mathrm{ha}^{-1}$ na dose de $100 \mathrm{~kg} \mathrm{ha}^{-1} \mathrm{de}_{2} \mathrm{O}_{5}$ para a textura média, o uso da adubação reduz para $15,1 \%$ a diferença de produção entre os ambientes. A diferença na capacidade produtiva pode estar influenciada não só pela textura e níveis de fósforo no solo, mas também pelos níveis de outros atributos como os indicados por Dedecek et al. (2008), em que as maiores produtividades de Pinus taeda foram encontradas em solos que apresentaram maiores teores de potássio, fósforo, $\mathrm{pH}$, 
saturação de bases e menor saturação de alumínio.

Como as rotações de Pinus taeda são de longo prazo se faz necessário uma avaliação econômica com idade mais próxima do corte final para definição dos níveis efetivos de resposta e dosagens econômicas ótimas com o uso da adubação fosfatada.

\section{Conclusões}

O uso de adubação fosfatada promoveu ganhos significativos na produtividade de Pinus taeda até os sete anos de idade plantados em Latossolo Vermelho de textura média. A resposta se deu de forma linear com máxima produção para a dose de $100 \mathrm{~kg} \mathrm{ha}^{-1}$ de $\mathrm{P}_{2} \mathrm{O}_{5}$ adicionados logo após o plantio.

Para o Latossolo Vermelho de textura argilosa não houve resposta significativa ao uso de adubação fosfatada até os sete anos de idade.

\section{Referências Bibliográficas}

BELlOTE, A. F. J.; DEDECECK, R. A. Atributos físicos e químicos do solo e suas relações com o crescimento e a produtividade de Pinus taeda. Boletim de Pesquisa Florestal, Colombo, n. 53, p. 21 - 38, jul./dez. 2006.

BOOTH T. H.; JOVANOVIC, T. Improving descriptions of climatic requirements in the CABI Forestry Compendium. A report for the Australian Centre for International Agricultural Research. CSIRO - Forestry and Forest Products, Client Report $\mathrm{n}^{\circ}$. 758. 2000.

DEDECEK, R. A. et al. Influência do sítio no desenvolvimento do Pinus taeda aos 22 anos: 1. Características físico-hídricas e químicas do solo. Floresta, Curitiba, v. 38, n. 3, p. 507-516, jul/set. 2008.

FAUSTINO, L. I. et al. Crecimiento de cuatro familias de Pinus taeda en respuesta a la fertilización con nitrógeno y fósforo en el establecimiento de la plantación. Rev. Fac. Agron. Vol 111 (2): 54-63.201, 2012.

FERREIRA, C. et al. Nutrição de Pinus no sul do Brasil: diagnóstico e prioridades de pesquisa. Colombo: Documentos Embrapa Florestas, 2001. 23p.

FERNÁNDEZ, R. et al. Respuesta Del Pinus taeda y Ia Araucaria angustifolia a Ia adición de $\mathrm{N}, \mathrm{P}$ y $\mathrm{K}$ en Ia implantación. In: SILVOARGENTINA I, Governador Virasoro, Comentes, 2000. 1 CD-Rom.

FOX, T. R., JOKELA, E. J., ALLEN, H. L. The development of pine plantation silviculture in the southern United States. Journal of Forestry, 105(7), 337-347. 2007.

GATIBONI, L. C.; SILVA, L. S.; ANGHINONI, I. Diagnóstico da fertilidade do solo e recomendação da adubação. In: Leandro Souza da Silva; Luciano Colpo Gatiboni. (Org.). Manual de calagem e adubação para os estados do Rio Grande do Sul e de Santa Catarina. 11ed. Frederico Westphalen: SBCSNRS, 2016, v. 1, p. 89-99.

GONÇALVES, J.L.M. Recomendações de adubação para Eucalyptus, Pinus e espécies típicas da Mata Atlântica. ESALQ/USP. Documentos Florestais, v.15, p. 1-23. 1995.

HIGA, R.C.V. et al. Zoneamento climático: Pinus taeda no sul do Brasil. Colombo: Embrapa Florestas, 2008.

IBÁ - Indústria Brasileira de Árvores. Relatório IBA 2017. São Paulo. 2017

KRONKA, F.J.N.; BERTOLANI, F.; HERRERA PONCE, R. A cultura do Pinus no Brasil. São Paulo: SBS, 2005. 160 p.

MUNHOZ, J. S. B. Caracterização da produtividade florestal e dos padrões de crescimento de Pinus taeda L. no sul do Brasil através de análise de tronco. 2011. 116p 
Dissertação (Mestrado em Resursos Florestais) Escola Superior de Agricultura "Luiz de Queiroz”, Universidade de São Paulo, Piracicaba, 2011.

PRITCHETT, W. L.; ZWINFORD, K. R. Response of slash pine to colloidal phosphate fertilization. Soil Science of America Proceedings, Madison, v. 25, n. 5, p. 397-400, 1961.

REISSMANN, C.B.; WISNEWSKI, C. Aspectos nutricionais de plantios de Pinus. In: GONÇALVES, J.L.M.; BENEDETTI, V. Nutrição e fertilização florestal. Piracicaba: IPEF, 2005. cap. 5, p. 135-165.

RIGATTO, P. A.; DEDECEK, R. A.; MATTOS, J. L. M. Influência dos atributos do solo sobre a produtividade de Pinus taeda. Revista Árvore, Viçosa-MG, v.29, n.5, p.701- 709, 2005.

SAS. 2016 SAS Interprise Guide $\mathbf{7 . 1 2}$ for Windows Microsoft. SAS Institute Inc., Cary, USA, 2016

SCHULTZ, R.P. Loblolly pine: the ecology and culture of Loblolly pine (Pinus taeda L.). New Orleans: USDA, Forest Service, Southern Forest Experiment Station, 1997. 493p

TEDESCO, M. J. et al. Análise de solo, plantas e outros materiais. Porto Alegre: UFRGS, 1995. p. 174

VOGEL, H. L. M. et al. Crescimento inicial de Pinus taeda L. relacionado a doses de N, P e K. Ciência Florestal, Santa Maria, v. 15, n,2, p. 199-206, 2005. 